\title{
Ultrafast Time-Resolved Hard X-Ray Emission Spectroscopy on a Tabletop
}

\author{
Luis Miaja-Avila, ${ }^{1,}$ Galen C. O’Neil, ${ }^{1}$ Young I. Joe, ${ }^{1}$ Bradley K. Alpert, ${ }^{1}$ Niels H. Damrauer, ${ }^{2}$ William B. Doriese, ${ }^{1}$ \\ Steven M. Fatur, ${ }^{2}$ Joseph W. Fowler, ${ }^{1}$ Gene C. Hilton, ${ }^{1}$ Ralph Jimenez, ${ }^{2,3}$ Carl D. Reintsema, ${ }^{1}$ Daniel R. Schmidt, ${ }^{1}$ \\ Kevin L. Silverman, ${ }^{1}$ Daniel S. Swetz, ${ }^{1}$ Hideyuki Tatsuno, ${ }^{1}$ and Joel N. Ullom ${ }^{1,4, \dagger}$ \\ ${ }^{1}$ National Institute of Standards and Technology, 325 Broadway, Boulder, Colorado 80305, USA \\ ${ }^{2}$ Department of Chemistry and Biochemistry, University of Colorado Boulder, \\ Boulder, Colorado 80309, USA \\ ${ }^{3}$ JILA, National Institute of Standards and Technology and University of Colorado Boulder, \\ 440 UCB, Boulder, Colorado 80309, USA \\ ${ }^{4}$ Department of Physics, University of Colorado Boulder, Boulder, Colorado 80309, USA \\ (Received 28 March 2016; revised manuscript received 20 June 2016; published 27 September 2016)
}

\begin{abstract}
Experimental tools capable of monitoring both atomic and electronic structure on ultrafast (femtosecond to picosecond) time scales are needed for investigating photophysical processes fundamental to light harvesting, photocatalysis, energy and data storage, and optical display technologies. Time-resolved hard $\mathrm{x}$-ray $(>3 \mathrm{keV})$ spectroscopies have proven valuable for these measurements due to their elemental specificity and sensitivity to geometric and electronic structures. Here, we present the first tabletop apparatus capable of performing time-resolved x-ray emission spectroscopy. The time resolution of the apparatus is better than 6 ps. By combining a compact laser-driven plasma source with a highly efficient array of microcalorimeter x-ray detectors, we are able to observe photoinduced spin changes in an archetypal polypyridyl iron complex $\left[\mathrm{Fe}\left(2,2^{\prime} \text {-bipyridine }\right)_{3}\right]^{2+}$ and accurately measure the lifetime of the quintet spin state. Our results demonstrate that ultrafast hard $\mathrm{x}$-ray emission spectroscopy is no longer confined to large facilities and now can be performed in conventional laboratories with 10 times better time resolution than at synchrotrons. Our results are enabled, in part, by a 100- to 1000-fold increase in x-ray collection efficiency compared to current techniques.
\end{abstract}

DOI: 10.1103/PhysRevX.6.031047

\section{INTRODUCTION}

Molecular complexes and nanomaterials containing transition metals are being extensively investigated for light harvesting, photocatalysis, energy and data storage, and optical display technologies [1-5]. Many of these applications are inspired by the role of metal centers in photosynthetic light harvesting, charge migration, water oxidation, and hydrogen production. These technological and biological applications arise from the complex electronic structures of transition-metal compounds. For example, photoinduced electron transfer and multielectron chemistry are possible because of the existence of multiple stable oxidation states, whereas data storage and light emission are enabled by photoinduced and spontaneous

\footnotetext{
*Corresponding author. miaja@nist.gov

Corresponding author. joel.ullom@nist.gov

Published by the American Physical Society under the terms of the Creative Commons Attribution 3.0 License. Further distribution of this work must maintain attribution to the author(s) and the published article's title, journal citation, and DOI.
}

Subject Areas: Interdisciplinary Physics, Photonics, Physical Chemistry transitions between electron spin states, in spin-crossover and phosphorescent complexes, respectively.

The structure and dynamics of the chemical environment around the transition-metal atom have major effects on the energetics and lifetimes of accessible states and on the crossings between them. The lifetimes of spin- and chargetransfer states, which range widely from femtosecond to microsecond time scales, are primarily determined by the coupling among electronic and nuclear degrees of freedom. Major research efforts are focused on optimizing properties of transition-metal complexes for use in photochemical or photophysical applications [2,3]. Generalizing, there are two main issues under scrutiny. The first concerns the fate of the state prepared by photon absorption within the excited state manifold. Because of the high density of electronic and spin states of transition metals compared to purely organic molecules, relaxation processes almost always involve multiple steps with interconversion of states that are both charge transfer and ligand field in nature. For example, intersystem crossing involving several multiplicities-e.g., from singlet to quintet in $d^{6} \mathrm{Fe}$ (II) systems - may occur in the initial relaxation dynamics of a single complex. The second issue under scrutiny is tied to the issue of ground-state recovery, and involves questions 
of how much of the photon energy can be stored and for how long. Again, coupling to nuclear degrees of freedom, intersystem crossing events, and charge-transfer phenomena are central to both understanding and ultimately controlling excited state lifetimes.

Many of these issues have been confronted in the context of numerous studies of ruthenium(II) complexes for solar energy conversion. Part of their success is due to the energetic ordering of their excited states wherein the metalto-ligand charge-transfer (MLCT) manifold (both singlet and triplet) resides below ligand field excited states that can serve as significant nonradiative relaxation pathways. Lifetimes of approximately $1 \mu \mathrm{s}$ in room-temperature solvents are typical for the ${ }^{3} \mathrm{MLCT}$ of $\left[\mathrm{Ru}(\mathrm{bpy})_{3}\right]^{2+}$ (bpy $=2,2^{\prime}$-bipyridine) [6]. On the other hand, Fe(II) complexes have lower energy ligand field excited states leading to an extremely rapid deactivation of the MLCT states. As an example, the photoexcited ${ }^{1,3}$ MLCT manifold in $\left[\mathrm{Fe}(\text { bpy })_{3}\right]^{2+}$ is interconverted to a quintet metal center on subpicosecond time scales with significant loss of excited state energy that might otherwise be exploited for redox processes [7].

To ensure a productive interplay of experiment and theory in improving our fundamental understanding of metal complexes, the development of measurement techniques that probe the metal atom spin and electronic states on ultrafast time scales is a subject of intense focus. Traditionally, optical pump-probe spectroscopies have been used to study spin- and charge-transfer dynamics in metal complexes. However, changes in optical properties are often difficult to correlate with specific electronic or atomic configurations, and many of the relevant spin and electronic states are optically silent. In contrast, hard x-ray spectroscopy techniques, while much less accessible, are element specific and highly sensitive to both atomic and electronic structure [8]. One such technique, x-ray emission spectroscopy (XES), measures the spin and oxidation states of the absorbing atom while also being sensitive to electronic structure, local coordination, ligand type, and bond length $[9,10]$.

In nonresonant XES [see Fig. 1(a)], x-ray photons with energy greater than the binding energy of an inner-shell electron produce core-hole vacancies. These core holes are quickly filled by the relaxation of less tightly bound electrons with concomitant $\mathrm{x}$-ray fluorescence or emission $[9,10]$. The $K \alpha$ emission lines arise from $2 p$ electrons filling $1 s$ orbital vacancies, while the $K \beta_{1,3}$ and $K \beta^{\prime}$ emission lines result from $3 p \rightarrow 1 s$ transitions. Additionally, the $K \beta_{2,5}$ and $K \beta^{\prime \prime}$ lines result from transitions between the valence orbitals and $1 s$ core holes. The (a)

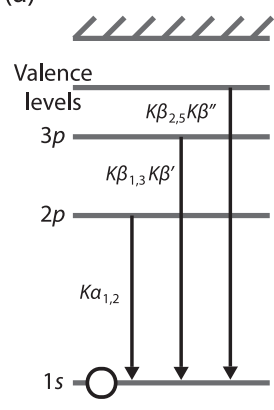

(b)

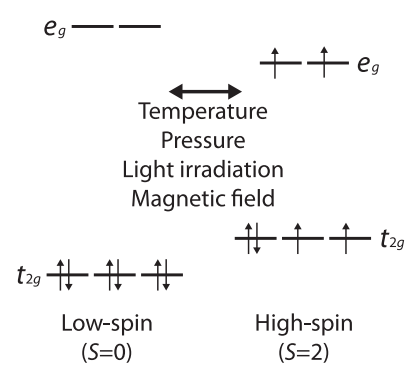

(c)

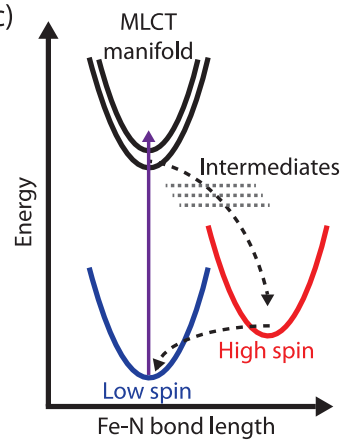

(d)

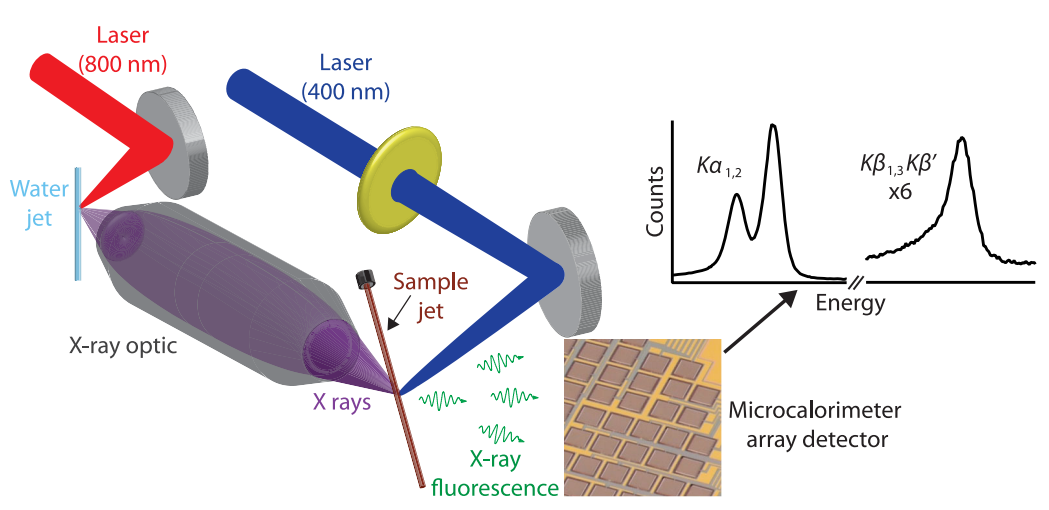

FIG. 1. (a) Energy-level diagram for x-ray emission after photoionization of a $1 s$ core electron. (b) Energy-level schematic showing low-spin and high-spin states of the $3 d$ orbital shell of Fe(II). (c) Schematic potential energy curves of Fe(II) complexes as a function of the Fe-N bond distance. (d) Experimental setup for time-resolved x-ray emission spectroscopy (TRXES) combining an ultrafast laser system, laser-driven $\mathrm{x}$-ray plasma source, sample jet, and array of microcalorimeter detectors. 
main $K \alpha$ and $K \beta$ lines are sensitive to the spin state of the valence electrons via an electronic exchange interaction. Both theoretical and experimental studies have shown that XES is an ideal tool to probe the spin states of complex molecular systems and crystalline or amorphous materials [11-16].

XES can thus be applied to the study of so-called spincrossover complexes. These molecules exhibit transitions between low-spin (LS) and high-spin (HS) states that can be induced by light, magnetic fields, temperature, or pressure [Fig. 1(b)]. Spin is an attractive state variable for information processing in molecular electronics [4], and knowing the time scale for spin crossover in low-spin $\mathrm{Fe}$ (II) complexes is important for their application in dyesensitized solar cells $[5,17]$. To study the dynamic behavior of spin states, time-resolved XES (TRXES) may be implemented in a "pump-probe" scheme, where an optical laser pulse first induces a spin transition in a sample and a subsequent $\mathrm{x}$-ray pulse probes the evolution of the spin state. The ultimate time resolution of these pump-probe techniques is set by the duration of the pump and probe pulses and the stability of their synchronization. Data collection is aided by intense exciting x-ray beams and detectors with excellent energy resolution that can collect a large fraction of the emitted $\mathrm{x}$ rays.

While ultrafast $x$-ray spectroscopies are uniformly challenging, TRXES is especially photon starved. As explained above, the signal of interest in XES is contained in secondary $\mathrm{x}$ rays fluoresced isotropically by the exciting $\mathrm{x}$-ray beam, rather than in perturbations to the exciting beam itself. Reliance on diverging secondary $x$ rays reduces the available signal by several orders of magnitude. The secondary $\mathrm{x}$-ray yield is proportional to the incident $\mathrm{x}$-ray flux, but short x-ray pulse durations are often achieved at the expense of reduced flux. The difficulty of TRXES is compounded by the need to measure the energy of the fluoresced photons with few-eV accuracy or better. Although crystal spectrometers can provide this level of accuracy, they are intrinsically inefficient. As a result of these challenges, TRXES measurements are currently restricted to a small number of large facilities because only these facilities provide exciting $\mathrm{x}$-ray pulses that are both sufficiently short and sufficiently intense. The time resolution of experiments performed at synchrotrons is set by characteristic electron-bunch lengths of 60-120 ps [18-22]. Slicing beam lines at synchrotrons produce $\sim 100$-fs $\mathrm{x}$-ray pulses and have been used to perform transient $x$-ray absorption measurements with subpicosecond time resolution [23,24]. However, the slicing process reduces the available $\mathrm{x}$-ray flux, and we are unaware of any TRXES measurements at sliced beam lines. TRXES experiments with time resolution better than $60 \mathrm{ps}$ have been performed only at the LCLS and SACLA free-electron laser facilities where sub-100-fs resolution is possible [7,25-28]. In the laboratory, static
XES measurements are possible [29-32], but measurements of ultrafast behavior have been stymied by the limited intensity of laboratory x-ray sources with suitable pulse duration and the limited collection efficiency of high-resolution x-ray spectrometers.

\section{EXPERIMENTAL SETUP}

Here, we present a tabletop apparatus capable of performing TRXES measurements with sub-6-ps time resolution. We use our apparatus to measure the spin dynamics in $\left[\mathrm{Fe}(\mathrm{bpy})_{3}\right]^{2+}$, an archetypal spin-crossover complex. To our knowledge, this is the first laboratory-based TRXES measurement and the only other TRXES installation, besides the ones at LCLS and SACLA, capable of obtaining sub-10-ps resolution. As shown in Fig. 1(d), our apparatus includes a Ti:sapphire laser with two output beams, a laser-driven x-ray plasma source, a polycapillary $\mathrm{x}$-ray optic, a sample jet, and an array of microcalorimeter detectors [33,34]. The 800-nm light from one laser output is focused onto a cylindrical water jet generating broadband bremsstrahlung $\mathrm{x}$-ray radiation. The polycapillary optic collects and refocuses $\mathrm{x}$ rays onto a nominal $80-\mu \mathrm{m}-$ diameter FWHM circular spot. The sample is a $50 \mathrm{mM}$ aqueous solution of $\left[\mathrm{Fe}(\mathrm{bpy})_{3}\right] \mathrm{Cl}_{2}$ in a $100 \mu$ m-diameter circular jet angled at $10^{\circ}$ with respect to the probing $\mathrm{x}$-ray beam. The sample is pumped with 400 -nm light generated by frequency doubling the second output of the Ti:sapphire laser. Details of the geometry where the pump, probe, and sample intersect are described in Appendix B and in Ref. [35]. Finally, the detector plane of the microcalorimeter array is positioned $75 \mathrm{~mm}$ from the sample and roughly $10^{\circ}$ out of the path of the probing $\mathrm{x}$ rays.

Hard $\mathrm{x}$-ray XES is typically performed with wavelengthdispersive $\mathrm{x}$-ray spectrometers that are based on Bragg diffraction [36,37]. When a spatially extended crystal is used to intercept more of the isotropically emitted $\mathrm{x}$ rays, the crystal must be curved to direct $\mathrm{x}$ rays of a given energy to a detection point. The pliability of individual crystals is finite and the use of many separate crystals poses an alignment challenge. As a result, there are limits to both the collecting area of crystal spectrometers and their ability to approach a point source. In contrast, our laboratory-based TRXES apparatus is based on an energy-resolving spectrometer with significantly different limits on collection efficiency and energy resolution. To compensate for the limited exciting flux from our laser plasma source, we use an array of 240 superconducting transition-edge microcalorimeters cooled to $0.115 \mathrm{~K}$ to suppress thermal and electrical noise [38]. The array provides an active detection area of $23 \mathrm{~mm}^{2}$, is located only $75 \mathrm{~mm}$ from the probed region of the sample, and provides few-eV energy resolution at hard $\mathrm{x}$-ray energies. Thus, it provides both a large collecting area and acceptable energy resolution. Additional details are given in Sec. IV C. The combination of a laser plasma source and microcalorimeter array has 
previously been proposed for time-resolved absorption spectroscopy [39] and used to obtain static absorption and emission spectra $[31,32,34,39]$, but the results we present here are the first demonstration of time-resolved $\mathrm{x}$-ray spectroscopy with this approach.

The measurements we describe here are performed on the molecular iron(II) complex $\left[\mathrm{Fe}(\mathrm{bpy})_{3}\right]^{2+}$, which undergoes metal-to-ligand charge transfer and a transition from a low-spin ground state to a high-spin excited state following the absorption of 400-nm light [7,18-20,40-44]. Spin crossover is accompanied by significant changes in molecular geometry [see Fig. 1(c)]. A recent TRXES measurement at the LCLS has provided evidence that after photoexcitation from the LS singlet state into MLCT states, $\left[\mathrm{Fe}(\mathrm{bpy})_{3}\right]^{2+}$ relaxes into the HS quintet state via a triplet intermediate [7]. Hence, $\left[\mathrm{Fe}(\mathrm{bpy})_{3}\right]^{2+}$ is an ideal system for the demonstration of in-laboratory TRXES.

\section{RESULTS}

Initially, we used our apparatus to measure the groundstate XES spectrum of $\left[\mathrm{Fe}(\mathrm{bpy})_{3}\right]^{2+}$ (see Fig. 2). The recorded XES spectrum is in excellent agreement with reference data [19] and includes the characteristic low-spin iron $K \alpha$ and $K \beta$ peaks in addition to a low-level background from the exciting $\mathrm{x}$-ray source. This background is due to photons from the x-ray source that scatter off air or the sample jet into the detector. Therefore, the spectral shape of the background matches the energy distribution of our broadband x-ray source. Figure 2 emphasizes the broadband response of microcalorimeter array detectors. Since we are not using any energy selecting or dispersing elements, e.g., Bragg crystals, the recorded spectrum contains $\mathrm{x}$ rays with energies from 3 up to $15 \mathrm{keV}$. The insets in Fig. 2 present a closer look at the $\mathrm{Fe} K \alpha$ and $K \beta$ spectral regions. We note that the $K \alpha$ and $K \beta$ spectra are acquired simultaneously and that emission lines from multiple elements in more complex compounds could also be acquired simultaneously. The energy resolution of the detector in the $K \alpha$ region is $5.2 \mathrm{eV}$, whereas the energy resolution in the $K \beta$ region is less well determined, but is near $5.5 \mathrm{eV}$.

Figure 3 shows both pumped and unpumped $\mathrm{Fe} K \alpha$ and Fe $K \beta$ emission spectra of aqueous $\left[\mathrm{Fe}(\mathrm{bpy})_{3}\right]^{2+}$ obtained with our apparatus. For the pumped measurement, the time delay between pump and probe is less than 6 ps. Figure 3 also shows fits that are used to determine the fraction of the sample that is in the high-spin state. We fit the spectra with a weighted sum of reference HS and LS spectra [19] convolved with a detector response function (see Appendix D for details). The pumped spectrum in Fig. 3(a) shows a decrease in the $K \alpha_{1}$ peak intensity compared to the unpumped spectrum. In Fig. 3(b), the pumped spectrum clearly shows the $K \beta_{1,3}$ peak decreasing in intensity and shifting to higher energy, while the weaker $K \beta^{\prime}$ feature becomes slightly more intense. These spectral changes are characteristic of a transition from LS to HS states [7].

Figure 4 shows the time evolution of the HS quintet fraction deduced from the $K \alpha$ and $K \beta$ spectral features measured at several time delays. Our data show a fast rise (see inset) of the HS fraction at time zero, followed by an exponential decay (see main figure) with a time constant of $570 \pm 100 \mathrm{ps}$. This time constant is consistent with the previously reported quintet lifetime of 665 ps $[19,43]$. When the $K \alpha$ and $K \beta$ data points are fit separately, the two results for the HS lifetime are statistically indistinguishable: $504 \pm 156$ and $612 \pm 117$ ps, respectively. Hence, the two line complexes provide equivalent information in this experiment. Additional details are provided in Appendix E.

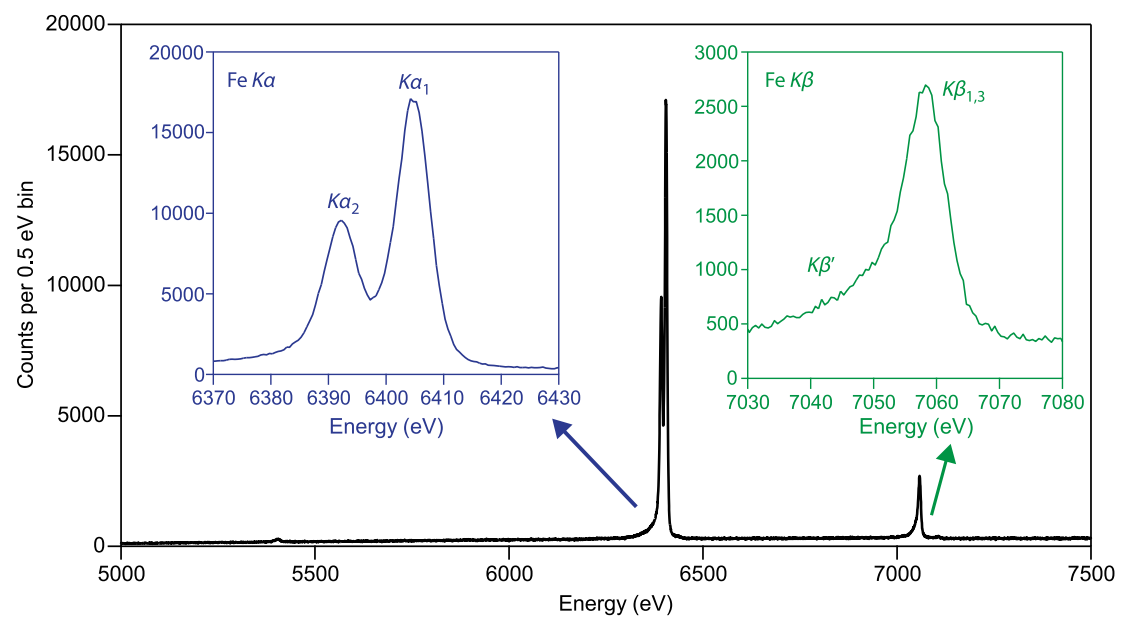

FIG. 2. X-ray emission spectra from $\left[\mathrm{Fe}(\mathrm{bpy})_{3}\right]^{2+}$. The XES features are characteristic of a low-spin Fe state and are in agreement with reference data [19]. The insets display the $K \alpha$ and $K \beta$ regions. The energy resolution of the detector in the $K \alpha$ region is $5.2 \mathrm{eV} \mathrm{FWHM.}$ The detector resolution in the $K \beta$ region is less well determined, but is near $5.5 \mathrm{eV}$. 

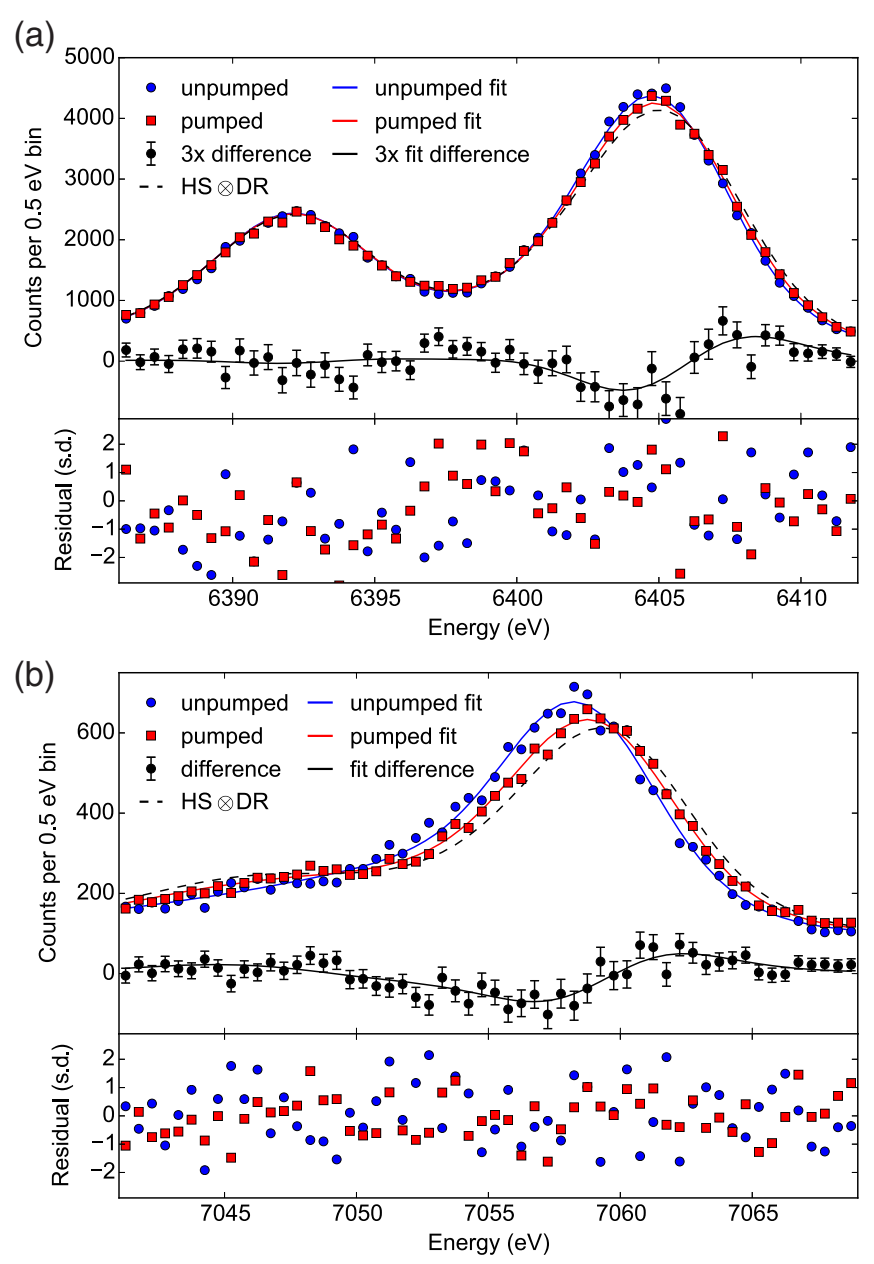

FIG. 3. X-ray emission spectra of $\left[\mathrm{Fe}(\mathrm{bpy})_{3}\right]^{2+}$ less than $6 \mathrm{ps}$ after photoexcitation. (a) $\mathrm{Fe} K \alpha$. (b) $\mathrm{Fe} K \beta$. Pumped and unpumped measurements are shown as red and blue markers, respectively. The dashed black lines are the reference high-spin spectra convolved with the detector response (DR) function. The solid red and blue curves are fits to the data that use a linear combination of high-spin and low-spin reference data convolved with the DR. The black markers show the difference between the pumped and unpumped data with 1-standard-deviation error bars. The solid black line is the difference between the two fit curves. The lower axes show residuals between the data and the two fit curves. Error values are calculated assuming Poisson statistics in each energy bin. This data set is acquired over $12.5 \mathrm{~h}$ of integration.

\section{DISCUSSION}

\section{A. Excluding the triplet state}

Recent work by Zhang et al. suggests that photoexcited $\left[\mathrm{Fe}(\mathrm{bpy})_{3}\right]^{2+}$ reaches the HS quintet state via a triplet intermediate [7]. When the pumped spectrum in Fig. 3 is fit to reference data for the triplet state rather than the quintet, we obtain an unphysical excitation fraction of $1.6 \pm 0.1$. Hence, we can exclude the presence of a substantial triplet population at this time in the photoreaction. The absence of a triplet population is consistent with the results of
Zhang et al., who found that evolution to the quintet state is complete on sub-ps time scales, which is faster than our expected time resolution.

\section{B. Time resolution}

The time resolution of our measurement is presently limited by three design choices made in favor of shortened integration times. First, the use of a high-efficiency polycapillary x-ray optic introduces a temporal broadening of $\sim 1.6$ ps due to internal path length differences. Second, we insert a fused silica rod in the pump path to increase the duration of the $400-\mathrm{nm}$ pulse from 40 fs to $1.3 \mathrm{ps}$. Longer pump pulses experience less nonlinear absorption and increase the fraction of sample converted to the high-spin state. Finally, the combination of orthogonal interaction between $\mathrm{x}$ rays and the pump beam and the size of the interaction region result in a temporal broadening of about $1.5 \mathrm{ps}$. The addition of these contributions in quadrature gives a predicted temporal resolution of $2.5 \mathrm{ps}$. To corroborate this calculation, we perform TRXES measurements with a temporal step size of 6 ps around time zero, as shown in the inset of Fig. 4. The transition from LS to HS states occurs between time steps, thus demonstrating time resolution better than $6 \mathrm{ps}$. Improvements in data collection should eliminate the need for the design compromises just described, thereby improving the time resolution. Ultimately, the fundamental limit on the temporal resolution is set by the duration of the pump and probe pulses. Before stretching, our 400-nm pump pulses are $40 \mathrm{fs}$, while $\mathrm{X}$-ray pulses as short as $100 \mathrm{fs}$ have been achieved using an

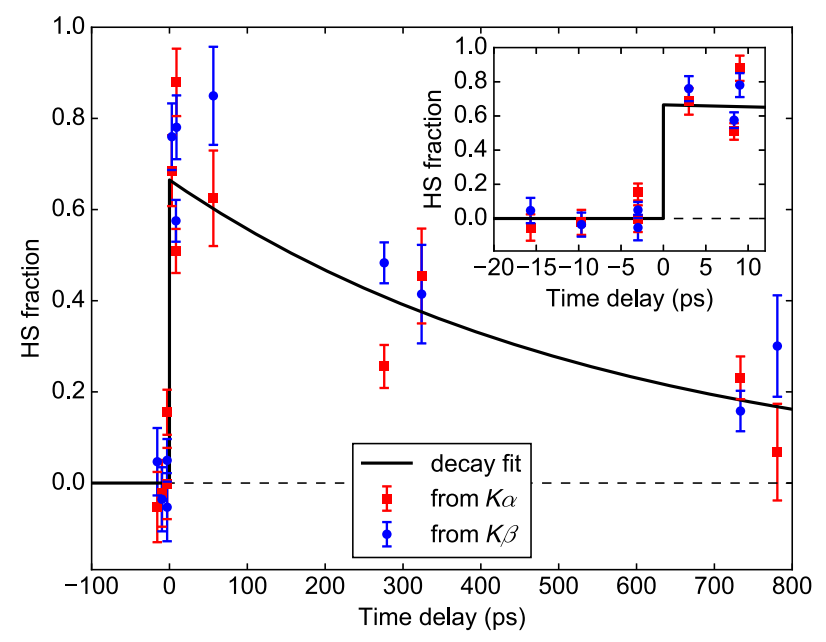

FIG. 4. Time evolution of high-spin (HS) fraction. Values for the HS fraction in optically excited $\left[\mathrm{Fe}(\mathrm{bpy})_{3}\right]^{2+}$ obtained from measurements of Fe $K \alpha$ and $K \beta$ spectra plotted versus time. A single-exponential fit to the HS fraction yields a decay-time constant of $570 \pm 100$ ps. Inset: Zoom into HS fraction at short time delays. Time zero is chosen to lie halfway between the two time points exhibiting the largest change in a measurement with 6-ps spacing between delays. These results show that our tabletop apparatus has a time resolution better than $6 \mathrm{ps}$. 
optimized laser plasma source [45]. Therefore, the potential exists for an order of magnitude improvement in experimental time resolution.

\section{Comparing the efficiency of photon usage to other measurements}

Here, we explore a metric for comparison of TRXES systems that is based on the efficiency of photon usage. In particular, we compare how accurately a TRXES system can measure the lifetime of a spin state with respect to the total number of $\mathrm{x}$-ray photons delivered to the sample. Haldrup et al. [19] recently reported using TRXES at beam line 7-ID of the Advanced Photon Source to measure the quintet lifetime of $\left[\mathrm{Fe}(\mathrm{bpy})_{3}\right]^{2+}$ to be $503 \pm 100 \mathrm{ps}$, with 95\% confidence limits. The total acquisition time was around $12 \mathrm{~h}$ and the exciting $\mathrm{x}$-ray flux was $2 \times 10^{12}$ photons/s. Consequently, the total number of photons delivered to the sample during the complete time-resolved measurement was approximately $8.6 \times 10^{16}$. In our own experiment, we measure the quintet lifetime of $\left[\mathrm{Fe}(\text { bpy })_{3}\right]^{2+}$ to be $570 \pm 100 \mathrm{ps}$, with $68 \%$ confidence. The total acquisition time is $191 \mathrm{~h}$ and the exciting $\mathrm{x}$-ray flux is $5 \times 10^{6}$ photons/s. Accordingly, the sample is exposed to a total of $3.4 \times 10^{12}$ photons. It can be seen that our results are achieved with a photon dose that is a factor of $2.5 \times 10^{4}$ lower, an advantage that could be critical for samples that are more radiation sensitive than $\left[\mathrm{Fe}(\mathrm{bpy})_{3}\right]^{2+}$. Some differences between the two experiments should be considered to perform the fairest possible comparison of collection efficiencies. While the error figures in the two measurements are nearly identical, they correspond to different confidence levels. To reconcile the different confidence levels, the scaling of the error of the quintet lifetime with the number of delivered photons is needed. To determine this scaling, we conduct fits to subsets of the data shown in Fig. 4. This process suggests that the error scales as the number of counts to a power between -1 and -2 , and, to be conservative, we choose -2 . If we use this scaling law to estimate the number of exciting photons required to achieve a $\pm 100-p s, 95 \%$ confidence level measurement of the quintet lifetime using our apparatus, then a factor of 4 more photons are needed in our measurement. In addition, the sample jet configuration in our experiment is more favorable for producing emission $\mathrm{x}$ rays. The sample concentration and interaction length between the sample and $\mathrm{x}$-ray beam in the work we present here (and in Ref. [19]) are $50 \mathrm{mM}(20 \mathrm{mM})$ and $450 \mu \mathrm{m}$ $(140 \mu \mathrm{m})$, respectively. These differences increase the absorption of the exciting x-ray beam and the production of emission $x$ rays by a factor of 13.5 in our work. The monoenergetic incident beam used by Haldrup is more efficient at creating $K$-shell vacancies than the broadband source we use in our work. Only $70 \%$ of the $x$ rays delivered to the sample in our experiment have sufficient energy to produce $K$-shell x-ray emission. Accounting for the various factors above, we estimate that the advantage in collecting efficiency of our apparatus over the work of Ref. [19] is $2.5 \times 10^{4} / 4 / 13.5 / 0.7=660$. This striking improvement explains how our apparatus has enabled TRXES on a table top.

It is useful to compare the factor of 660 advantage in photon collecting efficiency determined above to firstprinciples estimates based on detector properties such as solid angle and detection probability. Uhlig et al. [32] performed a detailed comparison between microcalorimeter arrays and wavelength-dispersive spectrometers like the ones currently used at synchrotron and free-electron laser facilities. In this analysis, the $\mathrm{x}$-ray collecting efficiency of a microcalorimeter array similar to the one we use here was estimated to be 170 and 730 times larger than two modern spectrometers with 16 and 5 dispersive crystals, respectively. Uhlig et al. assumed a 20-mm distance between the sample and the microcalorimeter array, whereas the distance in our experiment is $75 \mathrm{~mm}$. While the increased distance reduces the collecting efficiency of the microcalorimeter by a factor of 14 , this effect is essentially balanced by attenuation of the emitted photons within the wavelength-dispersive instruments. For generality, this attenuation was not included in the estimates of Uhlig et al. since it is small at $\mathrm{x}$-ray energies above $\sim 13 \mathrm{keV}$, but it is significant at the $6.5-7 \mathrm{keV}$ energies relevant here, even when a helium bag is used to reduce photon losses [36]. The spectrometer used in the work of Haldrup et al. [19] contained a single crystal analyzer that subtends $0.05 \%$ of $4 \pi$ sr [22]. Adapting the analysis of Uhlig et al., the predicted collecting efficiency advantage of the microcalorimeter instrument compared to this single crystal analyzer is a factor of almost 4600. Given the complexity of comparing such different experimental approaches, agreement to within an order of magnitude with the factor of 660 described in the previous paragraph is encouraging. To summarize this discussion, our results show that we can determine spin-state lifetimes with similar precision to measurements conducted at large facilities but using radiation doses to the sample that are between 2 and 3 orders of magnitude smaller.

\section{Impacted areas of science}

Radiation damage alters the material under study and is a crucial issue when $\mathrm{x}$ rays are used to probe biological materials [46]. The utility of ever-brighter $x$-ray sources depends on the development of techniques to mitigate or avoid such damage. Proven techniques include cooling samples to cryogenic temperatures, rapid substitution of fresh sample material, and the use of x-ray pulses whose termination precedes the onset of damage [47]. These efforts not withstanding, overcoming radiation damage remains a critical challenge, with the heavily studied and biologically critical metalloprotein photosystem II 
providing a particularly salient example [48]. Radiation damage is especially likely in XES and TRXES because a large number of exciting $\mathrm{x}$ rays are needed to produce the smaller number of isotropically emitted $\mathrm{x}$ rays that comprise the signal of interest. Improving the efficiency with which emitted $\mathrm{x}$ rays are collected reduces the number of $\mathrm{x}$ rays that need to be delivered to the sample and, thus, is another technique for reducing radiation damage. As we describe above, the collecting efficiency of our x-ray spectrometer significantly improves on the state of the art. This improvement may ease the study of damage-prone materials in a range of measurement scenarios and especially for so-called photon-in, photon-out spectroscopies such as XES, partial fluorescence yield x-ray absorption spectroscopy, and resonant inelastic $\mathrm{X}$-ray spectroscopy, in which the signal of interest is contained in secondary $\mathrm{x}$ rays produced by the probing $x$-ray beam. Improvements in photon collection efficiency are also beneficial for the study of trace or dilute specimens, where a future goal is the study of biological materials at in vivo concentrations.

In the Introduction, we note the large number of studies on ruthenium complexes for solar energy conversion. Nevertheless, due to the rarity and price of ruthenium, a number of research groups have focused on extending MLCT lifetimes in Fe(II) systems mainly by modifying the ligand structure. Duchanois et al. [49] and Harlang et al. [50] demonstrated highly efficient photoinduced electron injection from an $\mathrm{Fe}$ (II) molecular sensitizer into $\mathrm{TiO}_{2}$ by increasing the ligand field in the $\mathrm{Fe}(\mathrm{II})$ complex. Success in these experiments required $\mathrm{Fe}$ (II) systems where nonradiative decay from the photophysically nascent MLCT is slowed. Both groups reported record MLCT lifetimes of 16-18 ps. Additionally, Shepard et al. used steric bulk in ligand sets within bis-dihaloterpyrdine $\mathrm{Fe}$ (II) complexes to change the spin of the ground state and to achieve a 16-ps lifetime in the high-spin (quintet or septet) MLCT manifold [51]. Recently, Liu et al. reported observing a record excited state lifetime of $26 \mathrm{ps}$ for the ${ }^{3}$ MLCT state of an $\mathrm{Fe}$ (II) complex [52]. Interest in extending MLCT lifetimes is not confined to $\mathrm{Fe}$ (II) systems: Schrauben et al. increased the ${ }^{4} T_{2} \rightarrow{ }^{2} E$ intersystem crossing time in a Cr(III) system from 100 fs to 1.8 ps by exploring expanded tridentate ligands [53].

The large body of work targeting longer-lived molecular systems illustrates the need for a measurement technique sensitive to spin and metal oxidation state with a time resolution of a few ps. While x-ray free-electron laser (XFEL) facilities have the time resolution necessary for resolving intersystem crossing dynamics, there are presently only two facilities in the world with these capabilities, with very limited accessibility. There are also a small number of synchrotron facilities with beam lines suitable for TRXES. Unfortunately, these beam lines have time resolutions of 60-100 ps, which prevents them from observing few-ps dynamics. In contrast, our TRXES apparatus is already well positioned to study intersystem crossing dynamics with time scales of a few ps, and further improvements are planned.

It should also be noted that longer time scales -100 ps to ns-remain important for characterizing dynamics in numerous metal-based systems, and our apparatus can readily access this domain. One area of interest involves systems where optical signatures that interrogate the metal center directly are weak. In this context, we can point to emissive lanthanide ion species whose photophysics can be exploited in sensing, lighting, and laser technologies [54-58]. Within such systems, phosphorescence involves radially contracted $4 f$-electron metal-centered states. Even without changing spin multiplicity, it is difficult to optically access the manifold of such states due to Laporte selection rules. To sensitize lanthanide ion phosphorescence, researchers rely on energy-transfer phenomena originating from optically addressable ligand or antenna systems. In cases where these energy-transfer partners are in close proximity, time constants can approach the few-ns to 100-ps regime. Of particular interest will be systems where transition-metal-containing chromophores comprise the antenna such that TRXES can simultaneously monitor the spin and oxidation state of the excitation energy donor at the same time as the lanthanide ion acceptor.

\section{E. Future prospects}

Reasonable improvements will greatly increase the capabilities of our apparatus. Laser plasma sources that use targets with higher atomic number than water produce substantially more $\mathrm{x}$ rays and therefore can excite more $\mathrm{x}$ rays. Sources with $\mathrm{Hg}$ [59], and $\mathrm{Cu}$ [60] targets, among others, produce $\mathrm{x}$-ray fluxes 10-1000 times larger than our source. Kilopixel microcalorimeter arrays will be achieved in the near future, and modest engineering changes will shrink the distance between sample and spectrometer. Conservatively, these improvements have the potential to provide at least an additional $10 \times$ reduction in count times together with improved temporal resolution. The energy resolution of the microcalorimeter spectrometer can also be improved, thereby providing more information per detected photon. In this work, we achieve 5.2-eV FWHM resolution, but the detector technology is still under rapid development. We have since demonstrated 2.6-eV FWHM resolution in a similar configuration [61]. Wavelength dispersive spectrometers designed for enhanced collection efficiency at the expense of spectral resolution are currently under development and may also be suitable for in-laboratory TRXES [30].

While this article reports the first tabletop apparatus for ultrafast TRXES, key subcomponents such as the driving laser, laser plasma sources, and millikelvin cryostats are already commercial products. Even microcalorimeter x-ray spectrometers are now commercially available, albeit at a substantially earlier stage of development than the 
instrument we use here [62]. We, therefore, anticipate the dissemination of similar measurement tools to other laboratory-based facilities.

Optimism about the prospects for tabletop, ultrafast x-ray spectroscopies based on techniques like those described here can coexist with appreciation for the capabilities of large $\mathrm{x}$-ray facilities. The $\mathrm{x}$-ray intensities and temporal resolution of $\mathrm{x}$-ray free-electron lasers are unmatched, so these large machines are the tools of choice for measurements at the frontiers of sensitivity and speed. At the same time, tabletop techniques can benefit work at large facilities. While several XFELs are under construction worldwide to supplement the two existing ones, beam time will likely remain precious and comparatively expensive well into the future. Further, the number of material systems that merit study is vast, spanning natural and engineered compounds as well as the dependencies of dynamic behavior on variables like solvent and concentration. If tabletop techniques can perform even a modest fraction of the many potential measurements, then a secondary but important benefit will be a contribution to the successful operation of XFELs by allowing the finite resource of beam time to be allocated to the most interesting and challenging problems. In addition, some technologies developed for tabletop x-ray science can also benefit science at large facilities. For example, microcalorimeter array detectors will bring the same improvements in photon collection efficiency described here to photon-starved measurements at synchrotrons and XFELs.

\section{CONCLUSIONS}

In summary, we demonstrate the first tabletop apparatus capable of performing ultrafast time-resolved x-ray emission spectroscopy. This advance is enabled, in part, by the use of a microcalorimeter $\mathrm{x}$-ray spectrometer that provides a 100- to 1000-fold improvement in x-ray collecting efficiency. We measure the quintet-state lifetime of $\left[\mathrm{Fe}(\text { bpy })_{3}\right]^{2+}$ to be $570 \pm 100 \mathrm{ps}$, a value that is in good agreement with prior work. The broadband response of our detection apparatus is novel and we use it to perform the first simultaneous measurement of multiple x-ray spectral features in a TRXES experiment. We find that the dynamic behavior of the Fe $K \alpha$ and $K \beta$ emission lines shows the same quintet lifetime within the statistical uncertainty of our measurements. Finally, we exclude the presence of a significant triplet population on few-ps time scales after photoexcitation. The sub-6-ps time resolution of our tabletop apparatus for time-resolved x-ray emission spectroscopy is superior to performance achieved at synchrotrons and is surpassed only by that of x-ray free-electron lasers. The characteristic size and power consumption of our apparatus are meters and kilowatts, whereas the same quantities for large x-ray facilities are hundreds of meters and megawatts. Our apparatus thus has the potential to allow more routine study of actuation pathways and time scales in spin-crossover materials.

\section{ACKNOWLEDGMENTS}

We gratefully acknowledge financial support from the NIST Innovations in Measurement Science Program and from the DOE Office of Basic Energy Sciences. We thank teaching assistant K. Spettel and the Spring 2015 CHEM 4021 class at the University of Colorado Boulder for help in the large-scale synthesis of the $\left[\mathrm{Fe}(\mathrm{bpy})_{3}\right]^{2+}$ sample. We thank the authors of Ref. [19] for providing LS and HS reference spectra. We thank J. Uhlig for encouragement and advice.

L. M.-A. and G. C. O. contributed equally to this work.

\section{APPENDIX A: EXPERIMENTAL SETUP AND PROCEDURES}

The laser system is a commercial Ti:sapphire amplifier delivering 800-nm, 35-fs, p-polarized pulses with $20 \mathrm{~mJ} /$ pulse at $1 \mathrm{kHz}$. We use a 60/40 beam splitter inside the laser enclosure to divide the beam into pump and probe arms. The probe arm is expanded with a 1:2 telescope and focused by a $90^{\circ}$ off-axis parabolic mirror with an effective focal length of $10 \mathrm{~cm}$. The $p$-polarized focused laser beam interacts at grazing incidence with a $100-\mu \mathrm{m}$-diameter water jet in a vacuum chamber under a pressure of 8-9 Torr [34]. The interaction between the laser pulse and the water jet produces a plasma close to the water surface. The electric field of the driving laser pulse accelerates electrons in the plasma, and when the energetic electrons encounter the water jet, $\mathrm{x}$ rays are generated. The $\mathrm{x}$ rays are emitted into a full $4 \pi$ solid angle, so we use a polycapillary x-ray optic to collect $\mathrm{x}$ rays from the source and refocus them onto the sample. The nominal focused spot is $80 \mu \mathrm{m}$, containing an approximate x-ray flux of $5 \times 10^{6}$ photons/s. To compensate for drifts in the pointing of the $800-\mathrm{nm}$ beam, the water jet chamber is shifted automatically to maintain a steady $\mathrm{x}$-ray flux. The $\mathrm{x}$-ray flux is measured using a commercial $\mathrm{x}$-ray CCD camera. The output of the CCD is routed to a computer where a software routine is active that drives a motorized stage that hosts the water jet. This feedback system maintains a stable x-ray flux over many hours.

The 800-nm pump beam path includes a computercontrolled delay stage and a mechanical chopper running at $500 \mathrm{~Hz}$. The pump beam is then doubled in frequency using a $200-\mu \mathrm{m}$-thick Beta Barium Borate (BBO) crystal. In order to increase the $400-\mathrm{nm}$ pulse duration and the sample excitation fraction, we use an antireflection-coated 15.5-cm-long fused silica rod. We use an 800-nm third-harmonic generation $\mathrm{BBO}$ crystal to perform a cross-correlation between the 800 -nm pulse and the time elongated $400-\mathrm{nm}$ pulse. Our measurement indicates that after traveling through the fused silica rod, the 400-nm pulse duration is $1.3 \mathrm{ps}$. The beam then passes through two 
orthogonal cylindrical lenses resulting in a final spot size of $1000 \times 300 \mu \mathrm{m}$ at the sample jet location. The energy of the pump beam is approximately $900 \mu \mathrm{J} /$ pulse. The $\mathrm{x}$-ray and 400-nm beams approach the sample jet orthogonal to each other. Since the x-ray probe pulses repeat at $1 \mathrm{kHz}$ and the 400-nm pump pulses repeat at $500 \mathrm{~Hz}$ due to the chopper, the apparatus provides alternating measurements of the sample's dynamic and quiescent behavior. A pickoff photodiode in the pump arm responds to the 400-nm pulses and the output of the photodiode is encoded in the microcalorimeter data stream so that $\mathrm{x}$-ray pulses are tagged as coming from the pumped or unpumped sample.

\section{APPENDIX B: SAMPLE JET AND INTERACTION REGION}

The sample is circulated in a self-contained loop using a commercial peristaltic pump. In order to access the sample with the pump and probe beams, we use a $100-\mu$ m-diameter free-space jet angled $10^{\circ}$ with respect to the cylindrical axis of the polycapillary x-ray optic. With this geometry, the $400-\mathrm{nm}$ beam encounters a $100-\mu \mathrm{m}$-diameter cylinder of the sample, while the x-ray beam copropagates with the sample and has an effective interaction length of $450 \mu \mathrm{m}$ (see Fig. 5). After $2 \mathrm{~cm}$ of free-space travel, the sample jet enters a drain tube and the sample solution is recirculated to the high-pressure side of the jet. The microcalorimeter array detector is located $75 \mathrm{~mm}$ away from the interaction (a)

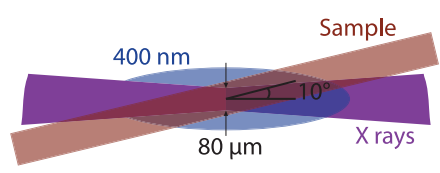

(b)

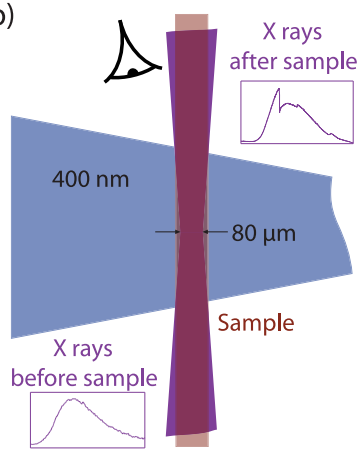

FIG. 5. X ray, pump, and sample interaction region. (a) Side view of the interaction region of the pump beam, X-ray beam, and the sample jet. The $100-\mu \mathrm{m}$-diameter sample jet is angled $10^{\circ}$ with respect to the central axis of the $\mathrm{x}$-ray beam. The $\mathrm{x}$-ray focus has a nominal diameter of $80 \mu \mathrm{m}$. The 400-nm pump beam is orthogonal to the other beams and therefore travels perpendicular to the plane of the page. The pump is focused using two orthogonal cylindrical lenses down to an ellipsoidal spot measuring $1000 \times 300 \mu \mathrm{m}$. (b) Top view of interaction region. Since the $10^{\circ}$ angle between $x$ rays and sample jet is measured with respect to the horizontal plane, the $\mathrm{x}$ rays and sample jet overlap in this view. The small insets show notional $\mathrm{x}$-ray spectra in photons versus energy before and after the sample jet. The sharp drop in the upper inset is due to the Fe $K$ absorption edge. The observer symbol denotes the orientation of the microcalorimeter array. region of the $\mathrm{x}$ rays, 400-nm light, and sample jet and is rotated roughly $10^{\circ}$ out of the path of the $\mathrm{x}$-ray beam so that it collects $\mathrm{x}$ rays emitted from the sample but not $\mathrm{x}$ rays from the exciting probe pulses.

\section{APPENDIX C: MICROCALORIMETER ARRAY DETECTOR AND PULSE ANALYSIS}

The microcalorimeter spectrometer consists of 240 transition-edge sensors, readout circuitry, and cryogenics to reach $75 \mathrm{mK}$. Each transition-edge sensor is a $350 \mu \mathrm{m} \times$ $350 \mu \mathrm{m}$ bilayer of molybdenum and copper with superconducting transition temperature $\sim 115 \mathrm{mK}$, and a $4.12-\mu \mathrm{m}-$ thick bismuth layer to increase the $\mathrm{x}$-ray stopping power. The sensors are voltage biased into the superconducting-tonormal transition where their resistance is a strong function of temperature and, hence, deposited energy. The absorption of an $\mathrm{x}$ ray results in a temperature change of $\sim 1 \mathrm{mK}$, causing a transient resistance increase and current decrease that recovers in a few ms. The magnitude of the current transient increases with energy and provides energy resolution. The current pulses are amplified by three stages of superconducting quantum interference devices (SQUIDs) configured in a time-division multiplexing architecture so that the output signals emerge from the spectrometer on eight signal lines. Pulse signals from $\mathrm{x}$-ray events are digitized, triggered, separated into discrete records of finite length, and stored at room temperature. At a later date, the record associated with each x-ray event is filtered and converted to energy. Cooling to $75 \mathrm{mK}$ is provided by a commercial adiabatic demagnetization refrigerator that requires no liquid cryogens. This type of spectrometer has recently been reviewed by Ullom and Bennett [38].

Data from each sensor are processed independently. To maximize the signal-to-noise ratio, an optimal filter is built and applied to each trace to arrive at a pulse height in arbitrary units [63]. The Fe $K \alpha$ and $K \beta$ complexes are identified and used to convert the arbitrary pulse heights to initial energy units. We regularly achieve about 5.2-eV FWHM energy resolution with this array at Fe $K \alpha$. The count rate per sensor is about 0.2 counts per second, which is well below the maximum counting rate of the instrument. Hence, use of a more intense $\mathrm{x}$-ray probe beam will straightforwardly shorten the required counting times. More information on the sensors and analysis techniques are given by Doriese et al. [61] and Fowler et al. [64]

\section{APPENDIX D: EXCITATION FRACTION CALCULATIONS FROM $K \alpha$ AND $K \beta$ SPECTRA}

The fraction of molecules in the high-spin state in a given spectrum is determined by maximum-likelihood [65] fitting to a model function $I(E)$ that describes the number of counts per unit of energy as a function of energy. The model functions are composed of a low-spin reference spectrum, a high-spin reference spectrum, and a detector 
response function. The reference spectra are the same as those used by Haldrup et al. [19], and were kindly provided to us by those authors. The intensity $I$ as a function of energy $E$ is

$$
I(E)=A\left[(1-f) \operatorname{LS}(E)+f \mathrm{HS}(E)+\frac{c}{A}\right] \otimes D R,
$$

where $A$ is the overall amplitude, $f$ is the fraction of molecules in the high-spin state, LS (HS) is the low- (high-) spin reference function, $c$ is a constant background, and $\otimes$ indicates convolution. The detector response function $\mathrm{DR}(E)$ is

$$
\mathrm{DR}(E)=e^{-E^{2} / 2 \sigma^{2}} \otimes\left[\frac{\chi}{\xi} e^{E / \xi} H(-E)+(1-\chi) \delta(E)\right],
$$

which consists of Gaussian broadening (where $2.355 \sigma$ is the FWHM energy resolution) and an exponential tail containing a fraction $\chi$ of all counts and with energy scale $\xi$. The undesirable exponential tail is the subject of current research, and is believed to be caused by the population of long-lived metastable states in the bismuth absorber. $H$ is the Heaviside function and $\delta$ is the Dirac delta function. When fitting single spectra, we allow for a possible energy shift $\Delta E_{0}$ and a possible linear stretch $\Theta$ in the energy calibration from its initial value. To minimize edge effects when convolving with our detector response model, we represent the reference data using sums of seven to nine Lorentzians following the procedure in Hölzer et al. [66].

It is useful to compare the peak excitation fraction of Fig. 4 to previous optical and x-ray measurements. For our pump-pulse energy and spot size, the fluence is $\sim 380 \mathrm{~mJ} / \mathrm{cm}^{2}$ and, on time scales short compared to the quintet lifetime, we observe an excitation fraction of $0.67 \pm 0.04$. The excitation fraction depends on sample concentration and geometry as well as the wavelength, fluence, and duration of the pump pulse. Excitation fractions have been published for several previous experiments $[7,19,67,68]$, but none share both the wavelength and pulse duration we use in this work. For a closer point of comparison, we perform separate optical transient absorption experiments on $\left[\mathrm{Fe}(\mathrm{bpy})_{3}\right]^{2+}$, wherein the ground-state optical bleach at $532 \mathrm{~nm}$ is used to deduce the excitation fraction. These optical transient absorption measurements are performed on a $100-\mu \mathrm{m}$-thick flat jet containing $28 \mathrm{mM}$ of $\left[\mathrm{Fe}(\mathrm{bpy})_{3}\right]^{2+}$. The 400 -nm pump pulses are chirped to about $700 \mathrm{fs}$, and, at the time of these experiments, the maximum achievable fluence was $230 \mathrm{~mJ} / \mathrm{cm}^{2}$. Under these conditions, we observe excitation fractions close to 0.45 . We also observe that the excitation fraction depends less than linearly on fluence at this fluence range, complicating extrapolation of the excitation fraction to the fluence values used in the x-ray experiments. After accounting for the higher sample concentrations in the x-ray experiments, the cylindrical sample jet, and the dependence of excitation fraction on fluence in an approximate fashion, we predict an excitation fraction slightly above 0.4 for the conditions of the x-ray experiment. One possible explanation for the difference between the excitation fraction observed in our x-ray data and the excitation fraction extrapolated from optical measurements is the difference in the optical pump durations: $1.3 \mathrm{ps}$ in the $\mathrm{x}$-ray experiment and $700 \mathrm{fs}$ in the optical measurements. A second possibility is the use of reference $\mathrm{x}$-ray spectra from the literature rather than curves measured with our own apparatus. Literature reference spectra reflect their own detector response function. For the spectra of Haldrup et al. [19] used here, the response function is described as a $1-\mathrm{eV}$ Gaussian. Any departure from this description will introduce bias to our fitting process. While we do not have access to the stable compound $\left[\mathrm{Fe}(\text { phen })_{2}(\mathrm{NCS})_{2}\right]$ used as a high-spin reference by Haldrup et al., acquiring microcalorimeter spectra of this material would be useful for future work.

\section{APPENDIX E: QUINTET-STATE LIFETIME CALCULATIONS}

The quintet lifetime and its error bar are determined by least-squares fitting the excitation fractions in Fig. 4 to the function

$$
f(t)=H\left(t-t_{0}\right) F_{0} e^{-\left(\left(t-t_{0}\right) / \tau\right)},
$$

where time zero $\left(t_{0}\right)$ is chosen to lie halfway between the two points exhibiting the largest change in a measurement with 6-ps spacing between delays, $F_{0}$ is the maximum excitation fraction, and $\tau$ is the lifetime of the quintet. Figure 4 includes results from every delay of every measurement cycle in which there are at least 15000 counts in the Fe $K \alpha$ complex. The total integration time is $191 \mathrm{~h}$.

[1] O. Kahn and C. J. Martinez, Spin-Transition Polymers: From Molecular Materials toward Memory Devices, Science 279, 44 (1998).

[2] A. Bousseksou, G. Molnár, L. Salmon, and W. Nicolazzi, Molecular Spin Crossover Phenomenon: Recent Achievements and Prospects, Chem. Soc. Rev. 40, 3313 (2011).

[3] M. Chergui, On the Interplay between Charge, Spin and Structural Dynamics in Transition Metal Complexes, Dalton Trans. 41, 13022 (2012).

[4] Does Molecular Electronics Compute?, Nat. Nanotechnol. 8, 377 (2013).

[5] W. Zhang and K. J. Gaffney, Mechanistic Studies of Photoinduced Spin Crossover and Electron Transfer in Inorganic Complexes, Acc. Chem. Res. 48, 1140 (2015).

[6] A. Juris, V. Balzani, F. Barigelletti, S. Campagna, P. Belser, and A. von Zelewsky, Ru(II) Polypyridine Complexes: 
Photophysics, Photochemistry, Eletrochemistry, and Chemiluminescence, Coord. Chem. Rev. 84, 85 (1988).

[7] W. Zhang et al., Tracking Excited-State Charge and Spin Dynamics in Iron Coordination Complexes, Nature (London) 509, 345 (2014).

[8] F. de Groot, High-Resolution X-Ray Emission, and X-Ray Absorption Spectroscopy, Chem. Rev. 101, 1779 (2001).

[9] P. Glatzel and U. Bergmann, High Resolution 1 s Core Hole X-Ray Spectroscopy in 3d Transition Metal ComplexesElectronic and Structural Information, Coord. Chem. Rev. 249, 65 (2005).

[10] U. Bergmann and P. Glatzel, X-Ray Emission Spectroscopy, Photosynth. Res. 102, 255 (2009).

[11] G. Vanko, T. Neisius, G. Molnár, F. Renz, S. Kárpáti, A. Shukla, and F. de Groot, Probing the 3d Spin Momentum with X-Ray Emission Spectroscopy: The Case of MolecularSpin Transitions, J. Phys. Chem. B 110, 11647 (2006).

[12] N. Lee, T. Petrenko, U. Bergmann, F. Neese, and S. Debeer, Probing Valence Orbital Composition with Iron $\mathrm{K} \beta X$-Ray Emission Spectroscopy, J. Am. Chem. Soc. 132, 9715 (2010).

[13] H. Gretarsson et al., Revealing the Dual Nature of Magnetism in Iron Pnictides and Iron Chalcogenides Using X-Ray Emission Spectroscopy, Phys. Rev. B 84, 100509 (2011).

[14] K. Tsutsumi, H. Nakamori, and K. Ichikawa, X-Ray Mn K $\beta$ Emission Spectra of Manganese Oxides and Manganates, Phys. Rev. B 13, 929 (1976).

[15] G. Peng, F. M. F. Degroot, K. Hamalainen, J. A. Moore, X. Wang, M. M. Grush, J. B. Hastings, D. P. Siddons, W. H. Armstrong, O. C. Mullins, and S. P. Cramer, HighResolution Manganese X-Ray Fluorescence Spectroscopy. Oxidation-State and Spin-State Sensitivity, J. Am. Chem. Soc. 116, 2914 (1994).

[16] M. Rovezzi and P. Glatzel, Hard X-Ray Emission Spectroscopy: A Powerful Tool for the Characterization of Magnetic Semiconductors, Semicond. Sci. Technol. 29, 023002 (2014).

[17] J. E. Monat and J. K. McCusker, Femtosecond Excited-State Dynamics of an Iron(II) Polypyridyl Solar Cell Sensitizer Model, J. Am. Chem. Soc. 122, 4092 (2000).

[18] G. Vankó, P. Glatzel, V.-T. Pham, R. Abela, D. Grolimund, C. N. Borca, S. L. Johnson, C. J. Milne, and C. Bressler, Picosecond Time-Resolved X-Ray Emission Spectroscopy: Ultrafast Spin-State Determination in an Iron Complex, Angew. Chem., Int. Ed. Engl. 49, 5910 (2010).

[19] K. Haldrup, G. Vankó, W. Gawelda, A. Galler, G. Doumy, A. M. March, E. P. Kanter, A. Bordage, A. Dohn, T. B. Van Driel, K. S. Kjær, H. T. Lemke, S. E. Canton, J. Uhlig, V. Sundström, L. Young, S. H. Southworth, M. M. Nielsen, and C. Bressler, Guest-Host Interactions Investigated by Time-Resolved X-Ray Spectroscopies and Scattering at $\mathrm{MHz}$ Rates: Solvation Dynamics and Photoinduced Spin Transition in Aqueous Fe(bipy) ${ }_{3}^{2+}$, J. Phys. Chem. A 116, 9878 (2012).

[20] G. Vanko et al., Spin-State Studies with XES and RIXS: From Static to Ultrafast, J. Electron Spectrosc. Relat. Phenom. 188, 166 (2013).

[21] C. Bressler, W. Gawelda, A. Galler, M. M. Nielsen, V. Sundström, G. Doumy, A. M. March, S. H. Southworth, L. Young, and G. Vankó, Solvation Dynamics Monitored by
Combined X-Ray Spectroscopies and Scattering: Photoinduced Spin Transition in Aqueous $\left[\mathrm{Fe}(\mathrm{bpy})_{3}\right]^{2+}$, Faraday Discuss. 171, 169 (2014).

[22] A. M. March, T. A. Assefa, C. Bressler, G. Doumy, A. Galler, W. Gawelda, E. P. Kanter, Z. Németh, M. Pápai, S. H. Southworth, L. Young, and G. Vankó, Feasibility of Valence-to-Core X-Ray Emission Spectroscopy for Tracking Transient Species, J. Phys. Chem. C 119, 14571 (2015).

[23] C. Bressler, C. Milne, V.-T. Pham, A. ElNahhas, R. M. van der Veen, W. Gawelda, S. Johnson, P. Beaud, and M. Chergui, Femtosecond XANES Study of the Light-Induced Spin Crossover Dynamics in an Iron(II) Complex, Science 323, 489 (2009).

[24] N. Huse, H. Cho, K. Hong, L. Jamula, F. M. F. De Groot, T. K. Kim, J. K. McCusker, and R. W. Schoenlein, Femtosecond Soft X-Ray Spectroscopy of Solvated TransitionMetal Complexes: Deciphering the Interplay of Electronic and Structural Dynamics, J. Phys. Chem. Lett. 2, 880 (2011).

[25] J. Kern et al., Taking Snapshots of Photosynthetic Water Oxidation Using Femtosecond X-Ray Diffraction and Spectroscopy, Nat. Commun. 5, 4371 (2014).

[26] P. Wernet et al., Orbital-Specific Mapping of the Ligand Exchange Dynamics of $\mathrm{Fe}(\mathrm{CO})_{5}$ in Solution, Nature (London) 520, 78 (2015).

[27] R. Alonso-Mori, D. Sokaras, D. Zhu, T. Kroll, M. Chollet, Y. Feng, J. M. Glownia, J. Kern, H. T. Lemke, D. Nordlund, A. Robert, M. Sikorski, S. Song, T.-C. Weng, and U. Bergmann, Photon-in Photon-out Hard X-Ray Spectroscopy at the Linac Coherent Light Source, J. Synchrotron Radiat. 22, 612 (2015).

[28] S. E. Canton et al., Visualizing the Non-Equilibrium Dynamics of Photoinduced Intramolecular Electron Transfer with Femtosecond X-Ray Pulses, Nat. Commun. 6, 6359 (2015).

[29] G. T. Seidler, D. R. Mortensen, A. J. Remesnik, J. I. Pacold, N. A. Ball, N. Barry, M. Styczinski, and O. R. Hoidn, A Laboratory-Based Hard X-Ray Monochromator for High-Resolution X-Ray Emission Spectroscopy and X-Ray Absorption near Edge Structure Measurements, Rev. Sci. Instrum. 85, 113906 (2014).

[30] L. Anklamm, C. Schlesiger, W. Malzer, D. Grötzsch, M. Neitzel, and B. Kanngießer, A Novel von Hamos Spectrometer for Efficient X-Ray Emission Spectroscopy in the Laboratory, Rev. Sci. Instrum. 85, 053110 (2014).

[31] Y. I. Joe, G. C. O’Neil, L. Miaja-Avila, J. W. Fowler, R. Jimenez, K. L. Silverman, D. S. Swetz, and J. N. Ullom, Observation of Iron Spin-States Using Tabletop X-Ray Emission Spectroscopy and Microcalorimeter Sensors, J. Phys. B 49, 024003 (2016).

[32] J. Uhlig et al., High-Resolution X-Ray Emission Spectroscopy with Transition-Edge Sensors: Present Performance and Future Potential, J. Synchrotron Radiat. 22, 766 (2015).

[33] J. N. Ullom, J. A. Beall, W. B. Doriese, W. D. Duncan, L. Ferreira, G. C. Hilton, K. D. Irwin, C. D. Reintsema, and L. R. Vale, Optimized Transition-Edge X-Ray Microcalorimeter with $2.4 \mathrm{eV}$ Energy Resolution at $5.9 \mathrm{keV}$, Appl. Phys. Lett. 87, 194103 (2005). 
[34] L. Miaja-Avila, G. C. O’Neil, J. Uhlig, C. L. Cromer, M. L. Dowell, R. Jimenez, A. S. Hoover, K. L. Silverman, and J. N. Ullom, Laser Plasma X-Ray Source for Ultrafast TimeResolved X-Ray Absorption Spectroscopy, Struct. Dyn. 2, 024301 (2015).

[35] See Supplemental Material athttp://link.aps.org/supplemental/ 10.1103/PhysRevX.6.031047 for details of sample preparation, spatial and temporal overlap, and data analysis.

[36] E. Kleymenov, J. A. Van Bokhoven, C. David, P. Glatzel, M. Janousch, R. Alonso-Mori, M. Studer, M. Willimann, A. Bergamaschi, B. Henrich, and M. Nachtegaal, Five-Element Johann-Type X-Ray Emission Spectrometer with a SinglePhoton-Counting Pixel Detector, Rev. Sci. Instrum. 82, 065107 (2011).

[37] R. Alonso-Mori, J. Kern, D. Sokaras, T.-C. Weng, D. Nordlund, R. Tran, P. Montanez, J. Delor, V. K. Yachandra, J. Yano, and U. Bergmann, A Multi-Crystal Wavelength Dispersive X-Ray Spectrometer, Rev. Sci. Instrum. 83, 073114 (2012).

[38] J. N. Ullom and D. A. Bennett, Review of Superconducting Transition-Edge Sensors for X-Ray and Gamma-Ray Spectroscopy, Supercond. Sci. Technol. 28, 084003 (2015).

[39] J. Uhlig, W. Fullagar, J. N. Ullom, W. B. Doriese, J. W. Fowler, D. S. Swetz, N. Gador, S. E. Canton, K. Kinnunen, I. J. Maasilta, C. D. Reintsema, D. A. Bennett, L. R. Vale, G. C. Hilton, K. D. Irwin, D. R. Schmidt, and V. Sundström, Table-Top Ultrafast X-Ray Microcalorimeter Spectrometry for Molecular Structure, Phys. Rev. Lett. 110, 138302 (2013).

[40] C. Creutz, M. Chou, T. L. Netzel, M. Okumura, and N. Sutin, Lifetimes, Spectra, and Quenching of the Excited States of Polypyridine Complexes of Iron(II), Ruthenium(II), and Osmium(II), J. Am. Chem. Soc. 102, 1309 (1980).

[41] J. K. McCusker, K. N. Walda, R. C. Dunn, J. D. Simon, D. Magde, and D. N. Hendrickson, Subpicosecond 1MLCT $\rightarrow$ 5T2 Intersystem Crossing of Low-Spin Polypyridyl Ferrous Complexes, J. Am. Chem. Soc. 115, 298 (1993).

[42] W. Gawelda, A. Cannizzo, V. Pham, F. Van Mourik, C. Bressler, and M. Chergui, Ultrafast Nonadiabatic Dynamics of $\left[\mathrm{FeII}(\mathrm{bpy})_{3}\right]^{2+}$ in Solution, J. Am. Chem. Soc. 129, 8199 (2007).

[43] W. Gawelda, V.-T. Pham, M. Benfatto, Y. Zaushitsyn, M. Kaiser, D. Grolimund, S. Johnson, R. Abela, A. Hauser, C. Bressler, and M. Chergui, Structural Determination of a Short-Lived Excited Iron(II) Complex by Picosecond X-Ray Absorption Spectroscopy, Phys. Rev. Lett. 98, 057401 (2007).

[44] G. Auböck and M. Chergui, Sub-50-Fs Photoinduced Spin Crossover in $\left[\mathrm{Fe}(\mathrm{bpy})_{3}\right]^{2+}$, Nat. Chem. 7, 629 (2015).

[45] J. Weisshaupt, V. Juvé, M. Holtz, S. Ku, M. Woerner, T. Elsaesser, S. Ališauskas, A. Pugžlys, and A. Baltuška, High-Brightness Table-Top Hard X-Ray Source Driven by Sub-100-Femtosecond Mid-Infrared Pulses, Nat. Photonics 8, 927 (2014).

[46] M. Weik, R. B. G. Ravelli, G. Kryger, S. McSweeney, M. L. Raves, M. Harel, P. Gros, I. Silman, J. Kroon, and J. L. Sussman, Specific Chemical and Structural Damage to Proteins Produced by Synchrotron Radiation, Proc. Natl. Acad. Sci. U.S.A. 97, 623 (2000).
[47] H. N. Chapman et al., Femtosecond X-Ray Protein Nanocrystallography, Nature (London) 470, 73 (2011).

[48] J. Yano, J. Kern, K. D. Irrgang, M. J. Latimer, U. Bergmann, P. Glatzel, Y. Pushkar, J. Biesiadka, B. Loll, K. Sauer, J. Messinger, A. Zouni, and V. K. Yachandra, X-Ray Damage to the $\mathrm{Mn}_{4} \mathrm{Ca}$ Complex in Single Crystals of Photosystem II: A Case Study for Metalloprotein Crystallography, Proc. Natl. Acad. Sci. U.S.A. 102, 12047 (2005).

[49] T. Duchanois, T. Etienne, C. Cebrián, L. Liu, A. Monari, M. Beley, X. Assfeld, S. Haacke, and P. C. Gros, An Iron-Based Photosensitizer with Extended Excited-State Lifetime: Photophysical and Photovoltaic Properties, Eur. J. Inorg. Chem. 2015, 2469 (2015).

[50] T. C. B. Harlang, Y. Liu, O. Gordivska, L. A. Fredin, C. S. Ponseca, P. Huang, P. Chábera, K. S. Kjaer, H. Mateos, J. Uhlig, R. Lomoth, R. Wallenberg, S. Styring, P. Persson, V. Sundström, and K. Wärnmark, Iron Sensitizer Converts Light to Electrons with 92\% Yield, Nat. Chem. 7, 883 (2015).

[51] S. G. Shepard, S. M. Fatur, A. K. Rappé, and N. H. Damrauer, Highly Strained Iron(II) Polypyridines: Exploiting the Quintet Manifold to Extend the Lifetime of MLCT Excited States, J. Am. Chem. Soc. 138, 2949 (2016).

[52] L. Liu, T. Duchanois, T. Etienne, A. Monari, M. Beley, X. Assfeld, S. Haacke, and P. C. Gros, A New Record Excited State 3MLCT Lifetime for Metalorganic Iron(II) Complexes, Phys. Chem. Chem. Phys. 18, 12550 (2016).

[53] J. N. Schrauben, K. L. Dillman, W. F. Beck, and J. K. McCusker, Vibrational Coherence in the Excited State Dynamics of $\mathrm{Cr}(\mathrm{acac})_{3}$ : Probing the Reaction Coordinate for Ultrafast Intersystem Crossing, Chem. Sci. 1, 405 (2010).

[54] M. P. Tsvirko, G. F. Stelmakh, V. E. Pyatosin, K. N. Solovyov, T. F. Kachura, A. S. Piskarskas, and R. A. Gadonas, Fast Electronic Relaxation in Lanthanide Porphyrins, Chem. Phys. 106, 467 (1986).

[55] J.-C. G. Bunzli and C. Piguet, Taking Advantage of Luminescent Lanthanide Ions, Chem. Soc. Rev. 34, 1048 (2005).

[56] W. S. Perry, S. J. A. Pope, C. Allain, B. J. Coe, A. M. Kenwright, and S. Faulkner, Synthesis and Photophysical Properties of Kinetically Stable Complexes Containing a Lanthanide Ion and a Transition Metal Antenna Group, Dalton Trans. 39, 10974 (2010).

[57] F. Quochi, M. Saba, H. Artizzu, M. L. Mercuri, P. Deplano, A. Mura, and G. Bongiovanni, Ultrafast Dynamics of Intersystem Crossing and Resonance Energy Transfer in Er(III)-Quinolinolate Complexes, J. Phys. Chem. Lett. 1, 2733 (2010).

[58] D. T. Thielemann, M. Klinger, T. J. A. Wolf, Y. Lan, W. Wernsdorfer, M. Busse, P. W. Roesky, A. N. Unterreiner, A. K. Powell, P.C. Junk, and G. B. Deacon, Novel Lanthanide-Based Polymeric Chains and Corresponding Ultrafast Dynamics in Solution, Inorg. Chem. 50, 11990 (2011).

[59] C. Reich, C. M. Laperle, X. Li, B. Ahr, F. Benesch, and C. G. Rose-Petruck, Ultrafast X-Ray Pulses Emitted from a Liquid Mercury Laser Target, Opt. Lett. 32, 427 (2007).

[60] F. Zamponi, Z. Ansari, C. Korff Schmising, P. Rothhardt, N. Zhavoronkov, M. Woerner, T. Elsaesser, M. Bargheer, 
T. Trobitzsch-Ryll, and M. Haschke, Femtosecond Hard X-Ray Plasma Sources with a Kilohertz Repetition Rate, Appl. Phys. A 96, 51 (2009).

[61] W. B. Doriese, K. M. Morgan, D. A. Bennett, E. V. Denison, C. P. Fitzgerald, J. W. Fowler, J. D. Gard, J. P. Hays-Wehle, G. C. Hilton, K. D. Irwin, Y. I. Joe, J. A. B. Mates, G. C. O’Neil, C. D. Reintsema, N. O. Robbins, D. R. Schmidt, D. S. Swetz, H. Tatsuno, L. R. Vale, and J. N. Ullom, Developments in Time-Division Multiplexing of X-Ray Transition-Edge Sensors, J. Low Temp. Phys. 184, 389 (2016).

[62] R. Cantor and H. Naito, Practical X-Ray Spectrometry with Second-Generation Microcalorimeter Detectors, Microsc. Today 20, 38 (2012).

[63] B. K. Alpert, R. D. Horansky, D. A. Bennett, W. B. Doriese, J. W. Fowler, A. S. Hoover, M. W. Rabin, and J. N. Ullom,
Note: Operation of Gamma-Ray Microcalorimeters at Elevated Count Rates Using Filters with Constraints, Rev. Sci. Instrum. 84, 056107 (2013).

[64] J. W. Fowler, B. K. Alpert, W. B. Doriese, Y. I. Joe, G. C. O'Neil, J. N. Ullom, and D. S. Swetz, The Practice of Pulse Processing, J. Low Temp. Phys. 184, 374 (2016).

[65] J. W. Fowler, Maximum-Likelihood Fits to Histograms for Improved Parameter Estimation, J. Low Temp. Phys. 176, 414 (2014).

[66] G. Hölzer, M. Fritsch, M. Deutsch, J. Härtwig, and E. Förster, $K \alpha_{1,2}$ and $K \beta_{1,3} X$-Ray Emission Lines of the $3 d$ Transition Metals, Phys. Rev. A 56, 4554 (1997).

[67] W. Gawelda, Ph.D. thesis, Ecole Polytechnique Federale de Lausanne, 2006.

[68] F. Alves Lima, Ph.D. thesis, Ecole Polytechnique Federale de Lausanne, 2011. 